New windows on massive stars: asteroseismology, interferometry, and spectropolarimetry

Proceedings IAU Symposium No. 307, 2014

G. Meynet, C. Georgy, J. H. Groh \& Ph. Stee, eds.

(C) International Astronomical Union 2015 doi:10.1017/S1743921314006978

\title{
Angular Diameters of O- and B-type Stars
}

\author{
Kathryn Gordon, ${ }^{1}$ Douglas Gies ${ }^{1}$ and Gail Schaefer ${ }^{2}$ \\ ${ }^{1}$ Department of Physics and Astronomy, Georgia State University, Atlanta, GA 30302, USA \\ email: kgordon@chara.gsu.edu \\ ${ }^{2}$ The CHARA Array of Georgia State University, Mt. Wilson, CA 91023, USA
}

\begin{abstract}
We are observing a sample of 10 O-type stars and 60 B-type stars to determine angular diameters using the Center for High Angular Resolution Astronomy (CHARA) Array, the foremost optical long baseline interferometer in the world. Our goal is to establish accurate stellar parameters to test modern theories of stellar evolution that include rotation. We will combine our stellar angular diameter measurements with flux and line measurements from spectroscopy, projected rotational velocities, and distances to determine radius, effective temperature, luminosity, equatorial rotational velocity, and evolutionary mass. Knowing these properties will allow us to place the stars in a Hertzsprung-Russell diagram and obtain estimates for the age and evolutionary state.
\end{abstract}

Keywords. stars: fundamental parameters (classification, colors, luminosities, masses, radii, temperatures, etc.), techniques: interferometric

\section{The CHARA Array}

The CHARA Array is a state of the art optical/infrared interferometer operated by Georgia State University at the Mt. Wilson Observatory in California (ten Brummelaar et al. 2005). The Array consists of six 1-m diameter telescopes arranged in a Y configuration, and it provides the highest resolution of any telescope at visible and near-infrared wavelengths. It is capable of resolving details as small as 200 micro-arcseconds. The light from the telescopes is sent through light pipes across the mountain to be combined in the beam combining lab. Moving carts with mirrors in the lab allow the path length difference of the beams to be adjusted so that the interference fringes may be observed. There are six beam combiners in use with the Array allowing observations from the $R$-band with PAVO to the $H$ - and $K$-bands with CLASSIC and CLIMB.

\section{Fringe Visibility Data}

The PAVO beam combiner (Ireland et al. 2008) operates in the $R$-band and splits light up into several spectral channels with tiny prisms. Figure 1 shows example PAVO data of the B9 V star HD 11502 from three brackets of observations. The angular sizes and physical radii (from Hipparcos distances) are listed in the figure. A bracket consists of observations of a calibrator star, followed by the target, followed by another calibrator star. The ideal calibrator will be as small as possible so as to be unresolved, but still bright enough to be close in magnitude to the target. The data are fit to obtain an angular size measurement. The CLIMB beam combiner (Sturmann et al.

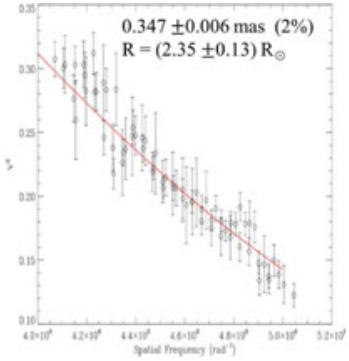

Figure 1. Fringe squared visibility data from PAVO of HD 11502. 
2010) operates in the $H$ - and $K$-bands and can use up to three telescopes at once. CLIMB has a resolution limit, operating on longest possible baselines, of about 0.5 mas in the $H$-band, while PAVO has a resolution limit of about 0.2 mas in the $R$-band.

\section{A New Companion for $\lambda$ Ori}

The O star $\lambda$ Ori currently has four known companions with the closest being about 4 arcsec away. Our visibility observations of $\lambda$ Ori show an oscillation in visibility with spatial frequency that is generally associated with the presence of a close binary companion. Preliminary fits of the visibility data indicate an angular separation of 1 to 3 mas which would put it at 0.3 to $1.0 \mathrm{AU}$ with a period of 10 to 54 days. These values are calculated using a distance of $324 \mathrm{pc}$ from Hipparcos and a typical mass of $23 M_{\odot}$ for an O 8 III star and $20 M_{\odot}$ for the companion (based on a flux ratio of $f_{2} / f_{1}=0.8$ ). Because we only have a single baseline of coverage for each night of observation, more modeling and observations with multiple baselines are necessary to further constrain the separation and orbit.

\section{Results and Future Work}

We currently have data on 25 stars from several observing runs at the CHARA Array, starting in 2012. More runs are planned through 2015. Priority is given to O stars and those B stars that have measured parallaxes in the Hipparcos catalog with errors less than $10 \%$ and/or that are members of clusters. We have closure phase data from CLIMB which will allow us to look for any light asymmetry caused by rotation or nearby companions. We also have good $(u, v)$ coverage from multiple baselines with CLIMB and PAVO that will allow us to detect an elliptical stellar shape caused by rapid rotation.

We obtain $T_{\text {eff }}$ by combining the angular diameter measurement with the integrated flux from the relationship $F_{\mathrm{bol}}=(1 / 4) \theta^{2} \sigma T_{\mathrm{eff}}^{4}$. The physical radii of our stars are found by combining the angular diameter with a known distance from parallax or cluster membership. Once $T_{\text {eff }}$ and the radius are known we can use a star's position in the H-R diagram to obtain mass and age estimates from theoretical evolutionary tracks. Our measurements of stellar properties, such as radius, temperature, mass, and age will provide fundamental reference data for nearby massive stars and will be of key importance for testing new stellar evolutionary models (Brott et al. 2011; Ekström et al. 2012).

\section{References}

Brott, I., de Mink, S. E., Cantiello, M., et al. 2011, A\&A 530, A115

Ekström, S., Georgy, C., Eggenberger, P., et al. 2012, A\&A 537, A146

Ireland, M. J., Mérand, A., ten Brummelaar, T. A., et al. 2008, in Society of Photo-Optical Instrumentation Engineers (SPIE) Conference Series, Vol. 7013 of Society of Photo-Optical Instrumentation Engineers (SPIE) Conference Series

Sturmann, J., ten Brummelaar, T., Sturmann, L., \& McAlister, H. A. 2010, in Society of PhotoOptical Instrumentation Engineers (SPIE) Conference Series, Vol. 7734 of Society of PhotoOptical Instrumentation Engineers (SPIE) Conference Series

ten Brummelaar, T. A., McAlister, H. A., Ridgway, S. T., et al. 2005, ApJ 628, 453 